\title{
Relación del clima motivacional creado por el entrenador con la motivación autodeterminada y la implicación hacia la práctica deportiva \\ Relationship between motivational climate created by coach regarding self-determined motivation and the involvement through the practice
}

\author{
David Sánchez-Oliva \\ Francisco Miguel Leo Marcos \\ Pedro Antonio Sánchez-Miguel \\ Diana Amado Alonso \\ Tomás García-Calvo \\ Facultad de Ciencias del Deporte. Universidad de Extremadura
}

\section{Resumen}

A través del presente estudio se pretendió valorar la relación existente entre el clima motivacional percibido por los jugadores y la motivación autodeterminada, y cómo éstos niveles de autodeterminación se relacionaban con la implicación mostrada por los jóvenes deportistas. Para ello, utilizamos una muestra compuesta por 97 jugadores de fútbol, con edades comprendidas entre los 12 y los 16 años, que completaron diversos cuestionarios encaminados a valorar las diferentes variables del estudio. Los resultados mostraron cómo el clima motivacional que implica a la tarea se relacionaba con los niveles altos de autodeterminación, así como con la satisfacción de las necesidades psicológicas básicas. Igualmente, comprobamos cómo la motivación autodeterminada se relacionaba positivamente con la implicación adecuada en el deporte. Como conclusión, destacamos la importancia por parte del entrenador de fomentar en el contexto deportivo un clima que implique a la tarea, con el objetivo de promover motivos de práctica intrínsecos a la propia actividad, lo que provocará una implicación más adecuada por parte de los deportistas.

Palabras clave: clima motivacional; mediadores psicológicos; motivación autodeterminada; implicación adecuada.

\begin{abstract}
This study aims to examine the relationships between motivational climate perceived by players and selfdetermined motivation, and how these self-determination levels are related with youth athletes' involvement. For instance, we have used a sample formed by 97 football players, ranging in age from 12 to 16 years old that completed questionnaires to assess different variables of the research. Results showed that mastery climate was associated with higher self-determination levels, as well as satisfaction of the psychological basic needs. Moreover, we demonstrated that self-determined motivation was positively related with an appropriate involvement in the sport. Finally, we emphasize the importance by coach to promote a mastery climate with the purpose to get greater intrinsic motives for practice, which leds to a more adequate involvement by athletes.
\end{abstract}

Key words: motivational climate; psychological needs; self-determined motivation; appropriate involvement. 
Sánchez-Oliva, D.; Leo, F. M.; Sánchez-Miguel, P. A.; Amado, D.; García-Calvo, T. (2010). Relación del clima motivacional creado por el entrenador con la motivación autodeterminada y la implicación hacia la práctica deportiva. Revista Internacional de Ciencias del Deporte, 20(6), 177-195.

\section{Introducción}

$\mathrm{D}$ urante la práctica deportiva en edad escolar, uno de los principales aspectos a fomentar por parte del entrenador debe ser el grado de implicación y compromiso que estos muestren en el contexto deportivo (Cecchini, González y Montero, 2007; García, Leo, Martín y Sánchez, 2008). En este sentido, se hace especialmente importante tener en cuenta la enorme influencia que pueden tener los factores sociales en los procesos psicológicos desarrollados por los jóvenes deportistas (Martín-Albo, Núñez y Navarro, 2003; Torregrosa, Cruz, Sousa, Viladrich, Villamarín, García-Mas y Palou, 2007), y la enorme relevancia que estas variables pueden tener en la implicación que muestren los jugadores adolescentes.

Como se expone en el modelo de la motivación intrínseca y extrínseca de Vallerand (2001), existen unos factores sociales que van a influir en el grado de satisfacción de las necesidades psicológicas básicas, produciendo mayores o menores niveles de autodeterminación. Dentro de los factores sociales, en nuestro trabajo adquieren especial relevancia los determinantes contextuales, ya que una de las variables estudiadas será el clima motivacional creado por el entrenador. Así, el clima motivacional percibido por los jugadores en la práctica deportiva influirá notablemente en el grado de satisfacción de las necesidades de autonomía, competencia y relaciones sociales (Vallerand, 2007).

El término clima motivacional fue introducido por Ames $(1984,1992)$ y Ames y Archer (1988) para designar los diferentes ambientes que crean los adultos significativos (padres, profesores, entrenadores) en los entornos de logro. En cuanto al clima motivacional del entrenador, Ames (1992) y Nicholls $(1989,1992)$ diferencian dos tipos. En primer lugar aparece el clima motivacional que implica a la tarea, donde se promueven valores como la diversión, satisfacción, interés y motivación intrínseca (Cervelló, Jiménez, Del Villar, Ramos y Santos-Rosa, 2004; Halliburton y Weiss, 2002; Theodosiou, Mantis y Papaioannou, 2008), donde el entrenador fomentará en sus jugadores el aprendizaje cooperativo y los jugadores estarán activamente implicados en el proceso de aprendizaje y en la elección de tareas (Cervelló et al., 2004; Cecchini, González, Carmona, Arruza, Escartí y Balagué, 2001; Ntoumanis, 2002). Por otro lado, encontramos el clima motivacional que implica al ego, que se encuentra relacionado positivamente con la orientación al ego, afectividad negativa y sentimientos de presión (Halliburton y Weiss, 2002; Theodosiou et al., 2008), donde los deportistas no pueden intervenir en las decisiones sobre el desarrollo de las sesiones de entrenamiento, las recompensas se establecen de manera pública y basadas en la demostración de un rendimiento superior y el entrenador destinará la mayor parte del tiempo a los niños que demuestren mayores niveles de habilidad (Cervelló et al., 2004; Theodosiou et al., 2008). 
Sánchez-Oliva, D.; Leo, F. M.; Sánchez-Miguel, P. A.; Amado, D.; García-Calvo, T. (2010). Relación del clima motivacional creado por el entrenador con la motivación autodeterminada y la implicación hacia la práctica deportiva. Revista Internacional de Ciencias del Deporte, 20(6), 177-195.

http://www.cafyd.com/REVISTA/02001.pdf

De este modo, el clima motivacional creado por el entrenador puede determinar el grado de satisfacción de las necesidades psicológicas básicas. Deci y Ryan (2000) consideran estas necesidades como nutrimentos psicológicos innatos que son esenciales para un prolongado crecimiento psicológico, integridad y bienestar. Así, estos autores identifican necesidades de autonomía, que se refiere al deseo de las personas de ser el origen de sus comportamientos, competencia, referido al deseo de las personas de sentirse competentes para producir resultados esperados e intentar prevenir los resultados no esperados, y relaciones sociales, que representa la sensación por parte del sujeto de una conexión con los individuos significativos del entorno social (Deci y Ryan, 2000; Ryan y Deci, 2000).

Igualmente, la percepción que cada sujeto tenga sobre los mediadores psicológicos van a influir de manera relevante en los niveles de autodeterminación. El nivel más alto de autodeterminación se denomina motivación intrínseca, y se refiere a la involucración en una actividad por propia iniciativa, por el placer y por la satisfacción que supone la participación en dicha actividad. Siguiendo en el continuo de motivación encontramos la motivación extrínseca, que se divide en tres constructos. De mayor a menor autodeterminación, en primer lugar aparece la regulación integrada, que aparece cuando un sujeto valora de manera positiva y beneficiosa una actividad, produciéndose una identificación con la misma y entendiendo el comportamiento por la apreciación por parte del sujeto de los beneficios que la actividad puede conllevar en su desarrollo personal. Posteriormente, la regulación introyectada, se asocia a sujetos que practican una actividad para evitar el sentimiento de culpabilidad y ansiedad, y así lograr mejoras de aspectos como el orgullo. Si proseguimos en el continuo de motivación, aparece la regulación externa, referida al compromiso por una actividad poco interesante para el sujeto, con el objetivo de conseguir una recompensa o evitar un castigo. Por último, el nivel más bajo de autodeterminación lo compone la desmotivación, que se caracteriza porque el sujeto no tiene intención de realizar una actividad, y por lo tanto, los comportamientos no han sido motivados ni intrínsecamente ni extrínsecamente (Deci y Ryan, 2000; Ryan y Deci, 2000).

En cuanto a los trabajos que han tratado de valorar estas variables, muchos estudios han comprobado las relaciones existentes entre las orientaciones motivacionales y los niveles de autodeterminación (Amorose, Anderson-Butcher, Flesch y Klinefelter, 2005; Moreno, Conte, Hellín, Hellín, Vera y Cervelló, 2008; Parish y Treasure, 2003).

Sin embargo, son menos los estudios encaminados por valorar las relaciones existentes entre el clima motivacional creado por el entrenador y los niveles de autodeterminación mostrados por los jugadores y cómo estas variables pueden predecir la implicación adecuada durante la práctica deportiva de los deportistas. 
Sánchez-Oliva, D.; Leo, F. M.; Sánchez-Miguel, P. A.; Amado, D.; García-Calvo, T. (2010). Relación del clima motivacional creado por el entrenador con la motivación autodeterminada y la implicación hacia la práctica deportiva. Revista Internacional de Ciencias del Deporte, 20(6), 177-195.

http://www.cafyd.com/REVISTA/02001.pdf

Sarrazin (2001) y Sarrazin; Vallerand, Guillet, Pelletier y Cury (2002), utilizando 335 jugadoras de balonmano con edades comprendidas entre 13 y 15 años, comprobaron la influencia positiva que el clima que implica a la tarea ejerce sobre la percepción autonomía, competencia y relaciones sociales, así como la relación negativa que el clima que implica al ego ejerce sobre la percepción de autonomía.

Igualmente, Standage, Duda y Ntoumanis (2003) llevaron a cabo un estudio en el contexto escolar utilizando una muestra compuesta por 328 estudiantes con edades comprendidas entre los 12 y los 14 años. Los resultados confirmaron cómo el clima motivacional que implica a la tarea se relacionaba de manera positiva y significativa con la satisfacción de las necesidades psicológicas básicas y con los mayores niveles de autodeterminación. Asimismo, aunque menos fuerte, estos autores también comprobaron la relación negativa entre el clima motivacional que implica al ego y la satisfacción de las necesidades psicológicas básicas. Por último, también destacaron la falta de relaciones significativas entre la desmotivación y el clima que implica al ego.

Entre los estudios realizados en nuestro país, cabe destacar los llevados a cabo por Cecchini y colaboradores. Así, Cecchini et al. (2004) utilizaron una muestra compuesta por 96 atletas con edades comprendidas entre los 14 y los 16 años, donde evidenciaron cómo los factores pertenecientes a la motivación intrínseca se asociaron positivamente con el clima que implica a la tarea y negativamente al clima que implica al ego. Resultados similares fueron obtenidos por Cecchini et al. (2005), donde utilizando jugadores jóvenes de fútbol demostraron cómo el clima que implica a la tarea se relacionaba positiva y significativamente con los motivos de práctica más intrínsecos.

Del mismo modo, Ntoumanis (2005) desarrolló un estudio con 460 estudiantes ingleses, con una edad media de 15 años, donde encontró relaciones positivas y significativas entre los dos tipos de climas y las 3 necesidades psicológicas básicas. En cuanto a los tipos de motivación, comprobó cómo el clima que implica a la tarea se relacionaba positivamente con la motivación intrínseca y con la regulación identificada, mientras que lo hacía de forma negativa con la desmotivación. Asimismo, este autor comprobó cómo el clima que implica al ego también se relacionaba positivamente con las motivaciones intrínseca, identificada e introyectada, mientras que lo hacía negativamente con la regulación externa y la desmotivación.

En un estudio posterior, Reinboth y Duda (2006) utilizaron 128 deportistas adolescentes, donde comprobaron las relaciones existentes entre el clima motivacional creado por el entrenador y satisfacción de las tres necesidades psicológicas básicas. Para ello, realizaron dos mediciones, al inicio y al final de la temporada, donde comprobaron cómo en ambos 
Sánchez-Oliva, D.; Leo, F. M.; Sánchez-Miguel, P. A.; Amado, D.; García-Calvo, T. (2010). Relación del clima motivacional creado por el entrenador con la motivación autodeterminada y la implicación hacia la práctica deportiva. Revista Internacional de Ciencias del Deporte, 20(6), 177-195.

http://www.cafyd.com/REVISTA/02001.pdf

periodos existía una relación positiva entre el clima motivacional que implica a la tarea y la satisfacción de las necesidades de competencia, autonomía y relaciones sociales.

Otro de los objetivos de este estudio era comprobar la relación que los diferentes niveles de autodeterminación y los mediadores guardan con la implicación que los jóvenes deportistas muestran en el contexto deportivo. Así, Reinboth, Duda y Ntoumanis (2004), con una muestra de 265 jugadores de fútbol y cricket adolescentes, comprobaron la relación existente entre la satisfacción de las necesidades psicológicas básicas y la implicación adecuada en el deporte (interés y satisfacción), mostrando la percepción de relaciones sociales la significatividad más elevada.

En este sentido, Chantal, Robin, Vernat y Bernache-Assollant (2005), realizaron dos estudios, el primero con una muestra de 102 sujetos y el segundo con 202, todos ellos jugadores federados en algún club deportivo y con edades comprendidas entre 18 y 24 años, en el que trataron de comprobar la importancia de la Teoría de la Autodeterminación para predecir y valorar diferentes aspectos deportivos y antideportivos en el deporte, entre los que se incluía la implicación de los jugadores. En el análisis de los resultados utilizaron un índice de autodeterminación, comprobando cómo los mayores índices de autodeterminación se asociaban positivamente con una adecuada implicación de los jóvenes jugadores.

Por último, Nickening, Fiorese, Pestillo y Lopes (2008) llevaron a cabo un estudio con 58 jugadores de voleibol, con una edad media de 19 años, diferenciando entre jugadores juveniles y jugadores adultos. Tras el análisis de sus resultados, no encontraron relaciones significativas entre los diferentes niveles de autodeterminación y la implicación adecuada en la práctica deportiva en los jugadores adultos. Sin embargo, en los jugadores juveniles, hallaron relaciones significativas entre la motivación intrínseca y la implicación adecuada que los jóvenes deportistas mostraron tanto en entrenamientos como en competición.

Así pues, el primer objetivo de este trabajo era comprobar cómo el clima motivacional creado por el entrenador se relacionaba con la satisfacción de las necesidades psicológicas básicas y los niveles de autodeterminación mostrados. Asimismo, nuestro segundo objetivo era establecer las relaciones existentes entre los niveles de autodeterminación y los mediadores psicológicos con la implicación en la práctica deportiva.

Igualmente, las hipótesis que guiaron nuestro trabajo fueron las siguientes. En primer lugar, estimamos que el clima que implica a la tarea se relacionaría positivamente con los mayores niveles de autodeterminación y con la satisfacción de las necesidades psicológicas básicas, mientras que el clima que implica al ego se asociaría con los menores niveles de autodeterminación. Del mismo modo, consideramos que los mayores niveles de 
Sánchez-Oliva, D.; Leo, F. M.; Sánchez-Miguel, P. A.; Amado, D.; García-Calvo, T. (2010). Relación del clima motivacional creado por el entrenador con la motivación autodeterminada y la implicación hacia la práctica deportiva. Revista Internacional de Ciencias del Deporte, 20(6), 177-195.

http://www.cafyd.com/REVISTA/02001.pdf

autodeterminación se asociarían positivamente con la implicación adecuada en la práctica deportiva.

\section{Método}

\section{Participantes}

La muestra de esta investigación estaba formada por 97 jugadores de fútbol, de género masculino y con edades comprendidas entre 12 y 16 años $(M=14.2 ; D T=1.23)$. Para seleccionar la muestra hemos utilizado el muestreo aleatorio por conglomerados. Todos los jugadores pertenecían a 12 equipos de las ligas local y provincial de la comunidad de Extremadura, donde 48 jugadores eran de categoría infantil y 49 de categoría cadete.

\section{Instrumentos}

Para la recogida de datos han sido utilizados diversos cuestionarios, que se especifican a continuación:

Clima motivacional de los entrenadores. Se utilizó la validación española realizada por Balaguer, Guivernau, Duda y Crespo (1997) del Perceived Motivational Climate in Sport Questionnaire (Newton y Duda, 1993; Newton, Duda y Yin, 2000). Dicha escala estaba precedida de una frase introductoria ("Tu entrenador..."), seguida de 21 ítems. Aplicamos los factores de segundo orden, que son el clima que implica al ego (9 ítems, ej.: "Motiva a los jugadores cuando juegan mejor que sus compañeros") y el clima que implica a la tarea (12 ítems, ej.: "Favorece que los jugadores se ayuden unos a otros en los entrenamientos y partidos"). En la versión española referida, aplicada a 216 jugadores de tenis, se realizó un Análisis Factorial Confirmatorio que refrendó la estructura dimensional del original. Se obtuvieron coeficientes de fiabilidad $\alpha$ de .80 para ambos factores. En la muestra específica de futbolistas empleada en este estudio, los coeficientes de fiabilidad han sido .89 para el clima que implica a la tarea y .78 para el clima que implica al ego.

Medida de la autodeterminación para la práctica deportiva. Se utilizó la Escala de Motivación en el Deporte (Pelletier, Fortier, Vallerand, Tudson, Brière y Blais, 1995), traducida al castellano por Núñez, Martín-Albo, Navarro y González (2006), en la que hemos adaptado la frase introductoria a la muestra de nuestra investigación. Así, este instrumento consta de una frase introductoria ("Yo practico fútbol..."), continuada por 28 ítems que miden las distintos motivos de práctica. Dicho cuestionario se divide en 5 factores, de los cuales, el primero se refiere a la motivación intrínseca (13 ítems, ej.: "Por la satisfacción que siento al aprender técnicas nuevas que nunca había realizado"). Otros 3 factores de este cuestionario están relacionados con la motivación extrínseca, dividida en motivación extrínseca identificada ( 3 ítems, ej.: "Porque es una de las mejores formas de mantener buenas relaciones con mis amigos"), introyectada (4 ítems, ej.: "Porque me sentiría mal si yo 
Sánchez-Oliva, D.; Leo, F. M.; Sánchez-Miguel, P. A.; Amado, D.; García-Calvo, T. (2010). Relación del clima motivacional creado por el entrenador con la motivación autodeterminada y la implicación hacia la práctica deportiva. Revista Internacional de Ciencias del Deporte, 20(6), 177-195.

http://www.cafyd.com/REVISTA/02001.pdf

no dedicase el tiempo necesario para practicarlo") y externa (4 ítems, ej.: "Porque me permite estar bien visto por la gente que conozco"). El último de los factores se denomina desmotivación (4 ítems, ej.: "No lo sé, tengo la impresión de que es inútil seguir practicando deporte"). La versión española anteriormente citada, se validó con una muestra de 275 deportistas pertenecientes a ocho deportes diferentes. El Análisis Factorial Confirmatorio, aportó una estructura de 7 factores, tres de motivación intrínseca, tres de regulación extrínseca y desmotivación. Sin embargo, en nuestro caso, hemos seguido las recomendaciones de diferentes autores (ver Deci y Ryan, 2000), que postulan que la variable motivación intrínseca solamente debe suponer un factor. Así, en este trabajo se han unido los tres tipos de motivación intrínseca en uno solo factor. En cuanto a la consistencia interna, se obtuvieron valores adecuados en todos los factores, oscilando los Alfa de Cronbach entre .70 y .80. En el presente estudio, la motivación intrínseca obtuvo un Alfa de Cronbach de .83, la regulación identificada .65 , la regulación introyectada .74 , la regulación externa .66 y la desmotivación .78.

Medida de las necesidades psicológicas básicas. Para valorar estas variables, se utilizaron las aportaciones de trabajos previos (Reinboth y Duda, 2006; Sarrazin et al., 2002, Standage et al., 2003), para adaptar al castellano los instrumentos utilizados y desarrollar la Escala de Mediadores Motivacionales (EMM: García, Sánchez, Leo, Sánchez y Gómez, 2009). Este instrumento consta de 18 ítems que fueron agrupados en 3 factores: autonomía (6 ítems, ej.: "Cuando juego a fútbol, me siento libre de poder expresar mis ideas y mis opiniones"), competencia (6 ítems, ej.: "Estoy satisfecho de mi rendimiento en el fútbol") y relaciones sociales (6 ítems, ej.: "Algunos de mis mejores amigos forman parte de este equipo"). Teniendo en cuenta que el instrumento no había sido validado previamente en población española, se realizó un Análisis Factorial Confirmatorio. Para ello se utilizó una muestra de 1897 jugadores de fútbol, baloncesto, balonmano y voleibol, con edades comprendidas entre 11 y 16 años $(M=12.37, D T=1.48)$. Se obtuvieron unos índices de ajuste aceptables: $\chi 2=$ $309.32, \mathrm{p}=.00 ; \chi 2 / \mathrm{g} .1 .=4.18 ; \mathrm{CFI}=.94 ; \mathrm{IFI}=.93 ; \mathrm{TLI}=.92 ; \mathrm{RMSEA}=.05 ; \mathrm{SRMR}=.03$. En cuanto a la consistencia interna, se obtuvieron valores $\alpha$ de .72 en la autonomía, .79 en la competencia y .76 en las relaciones sociales .76. En nuestro estudio, se refrendó la estructura obtenida en dicho análisis, obteniendo valores Alfa de Cronbach de .67 para el mediador autonomía, 79 para el mediador competencia y .73 para el mediador relaciones sociales.

Implicación adecuada en la práctica deportiva. Se ha empleado el factor implicación adecuada de la versión traducida y validada al castellano por Martín-Albo, Núñez, Navarro y González (2006) de la Escala Multidimensional de Orientaciones a la Deportividad (MSOS: Vallerand, Brière, Blanchard y Provencher, 1997), compuesto por 5 ítems (ej.: "Cuando compito, me entrego por completo aunque esté seguro de que vaya a perder"). En dicha 
Sánchez-Oliva, D.; Leo, F. M.; Sánchez-Miguel, P. A.; Amado, D.; García-Calvo, T. (2010). Relación del clima motivacional creado por el entrenador con la motivación autodeterminada y la implicación hacia la práctica deportiva. Revista Internacional de Ciencias del Deporte, 20(6), 177-195.

http://www.cafyd.com/REVISTA/02001.pdf

validación, estos autores realizaron un Análisis Factorial Confirmatorio, utilizando para ello 253 jugadores de diferentes deportes, donde obtuvieron un índice de fiabilidad en este factor de .71. En nuestro estudio, como se puede apreciar en la Tabla 1, se obtuvo un Alfa de Cronbach de .69 .

Como se puede comprobar, en varios factores se ha encontrado una fiabilidad inferior a .70. Sin embargo, eran valores muy cercanos, por lo que la validez interna puede ser aceptada debido al pequeño número de ítems que componen el factor (Hair, Anderson, Tatham y Black, 1998; Nunnally y Bernstein, 1995).

El formato de respuesta empleado en todos los instrumentos de la investigación es una escala tipo Likert en la que cada ítem tiene un rango de respuesta de 1 a 5 , donde 1 se correspondía con "totalmente en desacuerdo" y 5 con "totalmente de acuerdo" con la formulación de la pregunta.

\section{Procedimiento}

Para llevar a cabo la recogida de datos, se desarrolló un protocolo de actuación para que la obtención de datos fuera similar en todos los sujetos. El estudio se desarrolló durante los meses de Febrero y Octubre del año 2008. Se informó a los entrenadores sobre cuáles eran los objetivos de nuestra investigación y se pidió permiso a los padres indicándoles cuál iba a ser la utilización de los resultados. También se informó a los deportistas de que su participación era voluntaria y las respuestas serían tratadas confidencialmente. Los cuestionarios fueron rellenados por los jugadores durante 20 minutos antes del inicio del entrenamiento, estando el investigador presente para explicar el procedimiento y poder resolver las posibles dudas que surgieran entre los participantes.

\section{Resultados}

\section{Análisis descriptivo y correlacional}

En la Tabla 1, aparecen los valores descriptivos de cada una de las variables de la investigación. De esta forma, se observa la existencia de medias bastante elevadas en el clima que implica a la tarea, la motivación intrínseca, la percepción de autonomía y relaciones sociales y la implicación adecuada. Asimismo, la regulación identificada e introyectada y la percepción de competencia obtienen medias moderadamente altas, mientras que el clima que implica al ego y la desmotivación son los que poseen puntuaciones más bajas. 
Sánchez-Oliva, D.; Leo, F. M.; Sánchez-Miguel, P. A.; Amado, D.; García-Calvo, T. (2010). Relación del clima motivacional creado por el entrenador con la motivación autodeterminada y la implicación hacia la práctica deportiva. Revista Internacional de Ciencias del Deporte, 20(6), 177-195.

http://www.cafyd.com/REVISTA/02001.pdf

Tabla 1. Estadísticos descriptivos y Análisis de Fiabilidad.

\begin{tabular}{|c|c|c|c|}
\hline & $M$ & $D T$ & $\alpha$ \\
\hline Clima del entrenador & & & .89 \\
\hline Clima entrenador tarea & 4.09 & .68 & .78 \\
\hline Clima entrenador Ego & 2.41 & .75 & \\
\hline Motivaciones & & & .83 \\
\hline Intrínseca & 3.84 & .75 & .65 \\
\hline Identificada & 3.18 & 1.01 & .74 \\
\hline Introyectada & 3.58 & .81 & .66 \\
\hline Externa & 2.78 & .99 & .78 \\
\hline Desmotivación & 1.41 & .65 & .79 \\
\hline Mediadores & & & .67 \\
\hline Relaciones sociales & 3.96 & .69 & .79 \\
\hline Competencia & 3.55 & .69 & .79 \\
\hline Autonomía & 4.32 & & .79 \\
\hline Implicación adecuada & & & \\
\hline
\end{tabular}

Con el objetivo de comprobar las relaciones existentes entre los diferentes constructos de la Teoría de la Autodeterminación con el clima del entrenador y la implicación de los deportistas, realizamos un análisis de correlaciones bivariadas.

En la Tabla 2 se puede observar cómo el clima que implica a la tarea se relaciona de manera positiva y significativa con la motivación intrínseca, mientras que se asocia negativamente con la desmotivación. Asimismo, no se aprecian relaciones significativas entre este clima motivacional y alguna de las regulaciones pertenecientes a la motivación extrínseca. Por el contrario, se puede comprobar cómo el clima que implica al ego se relaciona de manera positiva y significativa con los niveles más bajos de autodeterminación, la regulación externa y la desmotivación, mientras que no aparecen relaciones destacables con los motivos de práctica más intrínsecos.

Asimismo, en cuanto a la relación que guarda el clima del entrenador percibido por los jugadores con los tres mediadores psicológicos, se observa cómo el clima que implica a la 
Sánchez-Oliva, D.; Leo, F. M.; Sánchez-Miguel, P. A.; Amado, D.; García-Calvo, T. (2010). Relación del clima motivacional creado por el entrenador con la motivación autodeterminada y la implicación hacia la práctica deportiva. Revista Internacional de Ciencias del Deporte, 20(6), 177-195.

http://www.cafyd.com/REVISTA/02001.pdf

tarea se correlaciona de forma positiva con las tres necesidades psicológicas básicas: relaciones sociales, competencia y autonomía, siendo la percepción de relaciones sociales el factor que obtuvo la relación más fuerte. En cambio, se puede apreciar cómo el clima que implica al ego tan sólo se relaciona significativamente con el sentimiento de autonomía, aunque el índice de correlación es bastante bajo.

Por último, con respecto a la implicación que los jóvenes jugadores muestran en la práctica deportiva, la motivación intrínseca se relaciona positiva y significativamente con la implicación adecuada. Igualmente, la regulación introyectada se asocia positivamente con la implicación de los jugadores, mientras que la desmotivación se asocia de manera negativa. En cuanto a los mediadores, la percepción de relaciones sociales, competencia y autonomía se correlacionan positivamente con la implicación adecuada en la práctica deportiva.

Tabla 2. Correlaciones bivariadas.

\begin{tabular}{|c|c|c|c|}
\hline & $\begin{array}{c}\text { Clima } \\
\text { Tarea }\end{array}$ & $\begin{array}{c}\text { Clima } \\
\text { Ego }\end{array}$ & $\begin{array}{c}\text { Implicación } \\
\text { Adecuada }\end{array}$ \\
\hline Motivaciones & & & \\
\hline Intrínseca & $.286\left(^{* *}\right)$ & .057 & $\left..381^{* *}\right)$ \\
\hline Identificada & .150 & .136 & .108 \\
\hline Introyectada & .141 & .152 & $.271\left(^{* *}\right)$ \\
\hline Externa & .083 & $.275\left(^{* *}\right)$ & .050 \\
\hline Desmotivación &.$-305\left(^{* *}\right)$ & $.245\left(^{* *}\right)$ & $-.263\left(^{* *}\right)$ \\
\hline Mediadores & & & $\left..355^{* *}\right)$ \\
\hline Relaciones sociales & $\left..605^{* *}\right)$ & -.110 & $.39\left(^{* *}\right)$ \\
\hline Competencia & $.325\left(^{* *}\right)$ & -.022 & $\left..333^{* *}\right)$ \\
\hline Autonomía & $.391\left(^{* *}\right)$ & $.204\left(^{*}\right)$ & \\
\hline
\end{tabular}

${ }^{* *} p<.01 ;{ }^{*} p<.05$

\section{Análisis de regresión lineal}

Se aplicó un análisis de regresión por pasos tomando como variable dependiente la implicación adecuada en la práctica deportiva, y como variables predictoras el resto de los diez factores medidos en nuestro estudio. Previamente, se comprobó la normalidad de los datos, obteniéndose en el índice de Asimetría valores comprendidos entre -0.7 y 0.9 , valores en el índice de Curtosis entre -0.8 y 0.7, así como puntuaciones adecuadas en el estadístico de 
Sánchez-Oliva, D.; Leo, F. M.; Sánchez-Miguel, P. A.; Amado, D.; García-Calvo, T. (2010). Relación del clima motivacional creado por el entrenador con la motivación autodeterminada y la implicación hacia la práctica deportiva. Revista Internacional de Ciencias del Deporte, 20(6), 177-195.

http://www.cafyd.com/REVISTA/02001.pdf

Kolmogorov-Smirnov $(d<.70 ; p>.10)$. Asimismo, el índice de tolerancia de las variables incluidas en la ecuación de regresión presentó valores comprendidos entre .81 y 1.00 , mientras que el índice FIV presentó valores entre 1.00 y 1.23 , por lo que la probabilidad de error derivada de la posible colinealidad queda descartada (Pérez, 2003).

Como se puede observar en los coeficientes de regresión, se obtuvo de forma global un porcentaje de varianza explicada del 33\%. En el primer paso, se obtuvo como mayor predictor la percepción de relaciones sociales, con un porcentaje de varianza explicada de $21 \%$. En el segundo paso, explicado con un $29 \%$ de varianza explicada, aparece como variable predictora la motivación intrínseca, mientras que en el tercer paso se incluye como variable predictora la percepción de competencia y obtiene un 33\% de varianza explicada. Como indica el análisis de regresión, tan sólo el primer paso obtiene una varianza superior al $10 \%$, por lo que los resultados correspondientes al paso 2 y 3 deberán tomarse con cautela.

Tabla 3. Análisis de regresión. Variable dependiente: Implicación adecuada.

\begin{tabular}{|c|c|c|c|c|c|}
\hline Variable & $\beta$ & $\mathrm{R}^{2}$ & $R^{2}$ ajustado & $\mathrm{t}$ & $\mathrm{P}$ \\
\hline Paso 1 & & .207 & .199 & & \\
\hline Relaciones sociales & .455 & & & 4.98 & .000 \\
\hline Paso 2 & & .285 & .270 & & \\
\hline Relaciones sociales & .385 & & & 4.29 & .000 \\
\hline Intrínseca & .288 & & & 3.20 & .002 \\
\hline Paso 3 & & .327 & .305 & & .002 \\
\hline Relaciones sociales & .302 & & & 3.20 & .004 \\
\hline Intrínseca & .263 & & & 2.98 & .018 \\
\hline Competencia & .225 & & & 2.4 & \\
\hline
\end{tabular}

\section{Discusión}

Como comentamos al inicio de este trabajo, el objetivo del mismo era analizar las relaciones que el clima del entrenador percibido por los jugadores tenía sobre los mediadores psicológicos y los niveles de autodeterminación, y cómo estas variables se relacionan con la implicación que tienen los jóvenes jugadores durante la práctica deportiva.

Si analizamos con detalle las relaciones existentes entre el clima motivacional percibido por los jugadores y los tipos de motivación mostrados por éstos, se puede observar cómo el clima 
Sánchez-Oliva, D.; Leo, F. M.; Sánchez-Miguel, P. A.; Amado, D.; García-Calvo, T. (2010). Relación del clima motivacional creado por el entrenador con la motivación autodeterminada y la implicación hacia la práctica deportiva. Revista Internacional de Ciencias del Deporte, 20(6), 177-195.

http://www.cafyd.com/REVISTA/02001.pdf

del entrenador que implica a la tarea se relaciona significativamente con la motivación intrínseca. Estos resultados también fueron hallados por otros autores (Cecchini et al., 2004; Cecchini et al., 2005; Ntoumanis, 2005; Standage et al., 2003), donde comprobaron cómo el clima que implica a la tarea provocaba motivos de práctica intrínsecos a la propia actividad. Así, parece razonable pensar que los jugadores que perciban un clima motivacional que implique a la tarea se correspondan con aquellos que muestren motivos de práctica más intrínsecos, ya que como ha sido demostrado en numerosos estudios (Moreno y Soledad, 2007; Reinboth y Duda, 2006; Standage, Duda y Pensgaard, 2005), un clima que implique a la tarea provoca una mayor percepción de autonomía, competencia y relaciones sociales, lo que se verá traducido en los mayores niveles de autodeterminación mostrados por los deportistas. Sin embargo, existes más discrepancias cuando hablamos de las relaciones existentes entre el clima que implica a la tarea y las diferentes regulaciones pertenecientes a la motivación extrínseca. De hecho, en nuestro estudio no hemos encontrado relaciones significativas entre el clima que implica a la tarea y alguna de las variables pertenecientes a la motivación extrínseca, mientras que Standage et al. (2003) comprobaron cómo el clima del entrenador que implica a la tarea se asociaba positivamente con la regulación introyectada. Igualmente, Ntoumanis (2005) halló relaciones positivas y significativas entre el clima que implica a la tarea y la regulación identificada. La razón de estas diferencias en los resultados puede deberse a las características de la muestra, ya que los dos estudios anteriormente comentados fueron realizados en el contexto educativo. A la vista de estos resultados, se hace necesaria la realización de más trabajos en el contexto deportivo encaminados a valorar la relación que existe entre los jugadores que perciben un clima que implica a la tarea y los jugadores que muestran motivos de práctica extrínsecos a la propia actividad.

Igualmente, al hablar del clima del entrenador que implica al ego se puede observar cómo esta variable se correlaciona significativamente con los motivos de práctica menos autodeterminados, la regulación externa y la desmotivación. Resultados similares fueron hallados por Cecchini et al. (2004), donde también comprobaron cómo los jugadores que percibían un clima que implica al ego se correspondían con aquellos que mostraban motivos de práctica menos autodeterminados. Sin embargo, estos resultados no coinciden con los encontrados con Standage et al. (2003), ya que no detectaron relaciones significativas entre el clima que implica al ego y los bajos niveles de autodeterminación. Asimismo, nuestros resultados no están en consonancia con los hallados por Ntoumanis (2005), donde comprobó como el clima que implica al ego se relacionaba negativa y significativamente con la regulación externa y la desmotivación. Por ello, teniendo en cuenta los estudios realizados, no podemos afirmar que la percepción de un clima que implique al ego se relacione con los motivos de práctica menos autodeterminados. 
Sánchez-Oliva, D.; Leo, F. M.; Sánchez-Miguel, P. A.; Amado, D.; García-Calvo, T. (2010). Relación del clima motivacional creado por el entrenador con la motivación autodeterminada y la implicación hacia la práctica deportiva. Revista Internacional de Ciencias del Deporte, 20(6), 177-195.

http://www.cafyd.com/REVISTA/02001.pdf

Por otro lado, debemos destacar la enorme influencia que tiene el clima del entrenador en la satisfacción de los mediadores psicológicos. Al igual que comprobaron otros autores (Reinboth y Duda, 2006; Sarrazin, 2001; Sarrazin et al. 2002; Standage et al., 2003), el clima del entrenador que implica a la tarea se relaciona de manera positiva y significativa con la percepción de autonomía, competencia y relaciones sociales. Así, si el entrenador fomenta en sus sesiones de entrenamiento valores como el progreso personal y el esfuerzo, conseguirá una mayor satisfacción de las tres necesidades psicológicas básicas. Igualmente, de manera inesperada, hemos obtenido una relación positiva entre el clima que implica al ego y el mediador autonomía, aunque los resultados se deben tomar con cautela, ya que el índice de correlación que presenta es bajo. Evidentemente, estos resultados no coinciden con los encontrados por otros autores, donde encontraron relaciones negativas del clima que implica al ego con la autonomía (Sarrazin, 2001; Sarrazin et al., 2002) y con las relaciones sociales (Reinboth y Duda, 2006). No cabe duda que de los tres mediadores, la percepción de competencia puede ser el que obtenga con mayor probabilidad una relación positiva con el clima que implique al ego, ya que los jugadores que perciban mayores niveles de habilidad pueden tender a aceptar este tipo de clima, donde continuamente se muestren comparaciones de rendimiento y desembocando en motivos de práctica extrínsecos a la propia actividad. A tenor de nuestros resultados, se ha comprobado cómo se cumple nuestra primera hipótesis, ya que se ha evidenciado la relación positiva que el clima que implica a la tarea guarda sobre la motivación intrínseca y la satisfacción de los mediadores psicológicos. Asimismo, se ha obtenido una asociación positiva entre el clima que implica al ego y los menores niveles de autodeterminación.

Cómo comentamos al inicio de este trabajo, uno de los objetivos del estudio era valorar la relación que existe entre la motivación autodeterminada y la implicación de los jóvenes jugadores durante la práctica deportiva. En cuanto a los niveles de autodeterminación, y comprobando cómo se cumple nuestra segunda hipótesis, se aprecia una relación positiva entre motivación intrínseca se asocia positivamente con la implicación adecuada de los jugadores, coincidiendo con los resultados obtenidos por otros estudios (Chantal et al., 2005; Nickening et al., 2008) donde comprobaron igualmente cómo los jugadores que mostraban motivos de práctica intrínsecos a la propia actividad se correspondían con aquellos que se implicaban positivamente en la práctica deportiva. Sin embargo, también se puede observar una relación positiva entre la regulación introyectada y la implicación adecuada de los jugadores en la práctica deportiva, hecho que no ocurre en otros estudios (Nickening et al., 2008), donde no encontraron relaciones significativas con alguna de las variables pertenecientes a la motivación extrínseca. La razón de esta relación con la regulación introyectada puede ser que teniendo en cuenta que los sujetos con este tipo de regulación practican deporte para evitar sentimientos de culpabilidad, estos jugadores también tendrán 
Sánchez-Oliva, D.; Leo, F. M.; Sánchez-Miguel, P. A.; Amado, D.; García-Calvo, T. (2010). Relación del clima motivacional creado por el entrenador con la motivación autodeterminada y la implicación hacia la práctica deportiva. Revista Internacional de Ciencias del Deporte, 20(6), 177-195.

http://www.cafyd.com/REVISTA/02001.pdf

altos niveles de compromiso e implicación en la práctica deportiva para evitar este tipo de sentimientos, es decir, sus comportamientos estarán influenciados en mayor medida por los pensamientos que puedan tener los compañeros del equipo. Asimismo, la razón de la falta de relaciones con la regulación identificada puede residir en que los jugadores con esta orientación motivacional practican deporte porque son conscientes de los beneficios que puede tener, pero los niveles de implicación durante la práctica deportiva no suelen ser los deseados.

En cuanto a los mediadores, se puede comprobar cómo la percepción de autonomía, competencia y relaciones sociales se relacionan de manera positiva y significativa con la implicación adecuada de los jóvenes deportistas. Estos resultados coinciden con los hallados por otros autores (Reinboth et al., 2004), donde además comprobaron cómo el mediador relaciones sociales era el que obtenía mayor índice de correlación, al igual que ocurre en nuestro estudio.

Al mismo tiempo, y atendiendo al análisis de regresión realizado, se aprecia cómo la motivación intrínseca y la percepción de competencia y relaciones sociales predicen significativamente la implicación adecuada, siendo el mediador relaciones sociales el que alcanza una mayor varianza explicada. Parece razonable pensar que los jugadores de un equipo que perciban mejores relaciones sociales entre los compañeros serán los que muestren un mayor grado de compromiso e implicación durante la práctica deportiva, como fue comprobado en otros estudios (García et al., 2008; Sousa et al., 2006).

Por lo tanto, la principal conclusión que podemos extraer de este estudio es la necesidad por parte de los entrenadores de fomentar y promover un clima que implique a la tarea, además de minimizar la aparición de un clima que implique al ego, hecho que provocará una mayor satisfacción de los mediadores y con ello niveles altos de autodeterminación. Asimismo, los motivos de práctica intrínsecos a la propia actividad provocarán una implicación más adecuada de los jóvenes jugadores durante la práctica deportiva.

Para conseguir fomentar un clima que implique a la tarea en el contexto deportivo, podemos utilizar diversas estrategias, como puede ser la aplicación del TARGET (Ames, 1992), acrónimo que representa las siguientes áreas: tarea, autoridad, recompensas, agrupación, evaluación y tiempo. En este sentido, autores como García (2006) indican también la necesidad de introducir objetivos a corto, medio y largo plazo para conseguir fomentar dicho clima. Del mismo modo, también resulta importante la puesta en marcha de programas de intervención con los principales significativos en la práctica deportiva de los jóvenes jugadores. Así, destaca la campaña desarrollada por García et al. (2009), dónde realizan charlas coloquios con padres y entrenadores en el contexto de la iniciación deportiva, con el objetivo de transmitir los valores positivos de la actividad física y deportiva y disminuir 
Sánchez-Oliva, D.; Leo, F. M.; Sánchez-Miguel, P. A.; Amado, D.; García-Calvo, T. (2010). Relación del clima motivacional creado por el entrenador con la motivación autodeterminada y la implicación hacia la práctica deportiva. Revista Internacional de Ciencias del Deporte, 20(6), 177-195.

http://www.cafyd.com/REVISTA/02001.pdf

comportamientos agresivos y antisociales. Con respecto a los entrenadores, se realizan talleres y dinámicas de grupo dónde se presentan las estrategias seguidas por Duda, Ntoumanis, Mahoney, Larson y Eccles (2005) para conseguir un clima que implique a la tarea en sus entrenamientos.

Por otro lado, del análisis de nuestros resultados se desprende la necesidad por parte del entrenador de fomentar buenas relaciones entre los miembros del grupo, ya que esto nos deparará una participación más satisfactoria por parte de los deportistas, aumentando con ellos la implicación que los jugadores muestren durante la práctica deportiva. Para ello, podemos recurrir a la propuesta realizada por Leo, Sánchez y García Calvo (2009), en la que se plantean diferentes estrategias a seguir con el objetivo de promover las relaciones sociales y la cohesión de los jugadores.

En cuanto a las limitaciones del estudio, podemos destacar que para medir el clima creado en el contexto deportivo utilizamos la percepción que tienen los jugadores, por lo que sería interesante contrastarlo con la percepción que tiene el propio entrenador sobre el clima creado. Al mismo tiempo, otra de las limitaciones del estudio es el tamaño de la muestra, lo que ha provocado índices de fiabilidad inferiores a los esperados. Por ello sería interesante utilizar una muestra más amplia en futuros trabajos, con el objetivo de analizar las diferencias en las variables estudiadas en función de la categoría o el deporte practicado.

\section{Referencias}

Ames, C. (1984). Competitive, cooperative, and individualistic goal structures: A cognitive-motivational analysis. En R. Ames y C. Ames (Eds.), Research on motivation in education: Vol.1. Student motivation (pp. 177-208). New York: Academic Press.

Ames, C. (1992). Achievement goals, motivational climate, and motivational processes. En G. C. Roberts (Ed.), Motivation in sport and exercise (pp. 161-176). Champaign, IL: Human Kinetics.

Ames, C., y Archer, J. (1988). Achievement goals in the classroom: Student's learning strategies a motivation processes. Journal of Educational Psychology, 80, 260-267.

Amorose, A. J.; Anderson-Butcher, D.; Flesch, S., y Klinefelter, L. (2005). Perceived motivational climate and self-determined motivation in male and female high school athletes. Research Quarterly for Exercise and Sport, 76, 96-97.

Balaguer, I.; Guivernau, M.; Duda, J. L., y Crespo M. (1997). Análisis de la validez de constructo y de la validez predictiva del cuestionario de clima motivacional percibido 
Sánchez-Oliva, D.; Leo, F. M.; Sánchez-Miguel, P. A.; Amado, D.; García-Calvo, T. (2010). Relación del clima motivacional creado por el entrenador con la motivación autodeterminada y la implicación hacia la práctica deportiva. Revista Internacional de Ciencias del Deporte, 20(6), 177-195.

http://www.cafyd.com/REVISTA/02001.pdf

en el deporte (PMCSQ-2) con tenistas españoles de competición. Revista de Psicología del Deporte, 11, 41-57.

Cecchini, J. A.; Carmona, A. M., y Contreras, O. R. (2004). Relaciones entre el clima motivacional, la orientación de meta, la motivación intrínseca, la auto-confianza, la ansiedad y el estado de ánimo en jóvenes deportistas. Psicothema, 16, 104-109.

Cecchini, J. A.; González, C., y Montero, J. (2007). Participación en el deporte y fair play. Psicothema, 19, 57-64.

Cecchini, J. A.; González, C.; Prado, J., y Brustad, R. J. (2005). Relación del clima motivacional percibido con la orientación de meta, la motivación intrínseca y las opiniones y conductas de fair play. Revista Mexicana de Psicología, 22(2), 469-479.

Cervelló, E.; Jiménez, R.; Del Villar, F.; Ramos, L., y Santos-Rosa, F. J. (2004). Goal orientations, motivational climate, equality, and discipline of spanish physical education students. Perceptual and Motor Skills, 99, 271-283.

Chantal, Y.; Robin, P.; Vernat, J. P., y Bernache-Assollant, I. (2005). Motivation sportspersonship and athletic aggression a mediational analysis. Psychology of Sport and Exercise, 6(2), 233-249.

Deci, E. L., y Ryan R. M. (2000). Target Article: The "What" and "Why" of Goal Pursuits: Human Needs and the Self-Determination of Behavior. Psychological Inquiry, 11(4), 227-268.

Duda, J. L.; Ntoumanis, N.; Mahoney, J. L.; Larson, R. W., y Eccles, J. S. (2005). Afterschool sport for children: Implications of a task-involving motivational climate. Mahwah, NJ, US: Lawrence Erlbaum Associates Publishers.

García-Calvo, T. (2006). Motivación y comportamientos adaptativos en jóvenes futbolistas. Tesis doctoral. Universidad de Extremadura.

García-Calvo, T.; Leo, F. M.; Martín, E., y Sánchez, P. A. (2008). El compromiso deportivo y su relación con factores disposicionales y situacionales contextuales de la motivación. Revista Internacional de Ciencias del Deporte, 12(4), 45-58.

García-Calvo, T.; Sánchez, P. A.; Leo, F. M.; Sánchez, D., y Gómez, F. R. (2009). Escuela del Deporte: Una Campaña para la promoción de los valores del deporte. Consejería de los Jóvenes y el Deporte. Junta de Extremadura.

García-Calvo, T.; Sánchez, P. A.; Leo, F. M.; Sánchez, D., y Gómez, F. R. (2009). Análisis psicométrico de la Escala de Mediadores Motivacionales (EMM). Manuscrito inédito. 
Sánchez-Oliva, D.; Leo, F. M.; Sánchez-Miguel, P. A.; Amado, D.; García-Calvo, T. (2010). Relación del clima motivacional creado por el entrenador con la motivación autodeterminada y la implicación hacia la práctica deportiva. Revista Internacional de Ciencias del Deporte, 20(6), 177-195.

http://www.cafyd.com/REVISTA/02001.pdf

Hair, J. F.; Anderson, R. E.; Tatham, R. L., y Black, W. C. (1998). Multivariate Data Analysis. Upper Saddle River, New Jersey: Prentice-Hall.

Leo, F. M.; García-Calvo, T.; Sánchez, P. A.; Gómez, F. R., y Sánchez, D. (2008). Relevancia de los climas motivacionales de los otros significativos sobre los comportamientos deportivos en jóvenes deportistas. Motricidad: European J ournal of Human Movement. 21, 119-136.

Leo, F. M.; Sánchez, P. A., y García, T. (2010). Mejora de la cohesión grupal a partir de tareas de entrenamiento en fútbol. Cienciaydeporte.net. Revista digital. En prensa.

Martín-Albo, J.; Núñez J. L.; Navarro, J. G., y González, V. M. (2006). Validación de la versión española de la escala multidimensional de orientaciones a la deportividad. Revista de Psicología del Deporte, 15(1), 9-22.

Martín-Albo, J.; Núñez, J. L., y Navarro, J. G. (2003). La evolución motivacional como criterio discriminante en los deportes. Revista Latinoamericana de Psicología, 35, 123.

Moreno, J. A.; Conte, L.; Hellín, P.; Hellín, G.; Vera, J. A., y Cervelló, E. (2008). Predicción de la motivación autodeterminada según las estrategias para mantener la disciplina y la orientación motivacional en estudiantes adolescentes de educación física. Apuntes de Psicología, 26 (3), 501-516.

Moreno, J. A., y Soledad, L. (2007). Predicción de la importancia concedida a la educación física según el clima motivacional y la motivación autodeterminada en estudiantes adolescentes. Enseñanza, 25, 137-155.

Moreno, J. A.; Cervelló, E., y González-Cutre, D. (2007). Analizando la motivación en el deporte: un estudio a través de la teoría de la autodeterminación. Apuntes de Psicología, 25(1), 35-51.

Newton, M., \& Duda, J. L. (1993). Elite adolescent athletes' achievement goals and beliefs concerning success in tennis. Journal of Sport and Exercise Psychology, 15, 437-448.

Newton, M.; Duda, J., \& Yin, Z. (2000). Examination of the psychometric properties of the Perceived Motivational Climate in Sport Questionnaire-2 in a sample of female athletes. J ournal of Sport Sciences, 18, 275-290.

Nicholls, J. G. (1989). The competitive ethos and democratic education. Cambridge, MA, US: Harvard University Press. 
Sánchez-Oliva, D.; Leo, F. M.; Sánchez-Miguel, P. A.; Amado, D.; García-Calvo, T. (2010). Relación del clima motivacional creado por el entrenador con la motivación autodeterminada y la implicación hacia la práctica deportiva. Revista Internacional de Ciencias del Deporte, 20(6), 177-195.

http://www.cafyd.com/REVISTA/02001.pdf

Nicholls, J. G. (1992). The general and the specific in the development and expression of achievement motivation. En G.C. Roberts (Ed.), Motivation in sport and exercise (pp.57-91). Champaign, IL: Human Kinetics.

Nickening, J. R.; Fiorese, L.; Pestillo, L., y Lopes, J. L. (2008). Motivação e atributos morais no esporte. Revista da Educação Física, 19(2), 173-182.

Ntoumanis, N. (2005). A Prospective study of participation in optional school physical education using a self-determination theory framework. Journal of Educational Psychology, 97(3), 444-453.

Nuñez, J. L.; Martín-Albo, J.; Navarro, J. G., y González, V. M. (2006). Preliminary Validation of a Spanish Version of the Sport Motivation Scale. Perceptual and Motor Skills, 102(3), 919-930.

Nunnally, J. C. y Bernstein, I. J. (1995). Teoría psicométrica. Madrid: McGraw-Hill.

Parish, L. E., \& Treasure, D. C. (2003). Physical activity and situational motivation in Physical Education: influence of the motivational climate and perceived ability. Research Quarterly for Exercise and Sport, 74, 173-182.

Pelletier, L. G.; Fortier, M. S.; Vallerand, R. J.; Tuson, K. M.; Brière, N. M., \& Blais, M. R. (1995). Toward a new measure of intrinsic motivation extrinsic motivation and amotivation in sports: The sport motivation scale (SMS). Journal of Sport and Exercise Psychology, 17, 35-53.

Pérez, C. (2003). Estadística. Problemas resueltos y aplicaciones. Prentice-Hall.

Reinboth, M., \& Duda, J. L. (2006). Perceived motivational climate, need satisfaction and indices of well-being in team sports: A longitudinal perspective. Psychology of Sport and Exercise, 7(3), 269-286.

Reinboth, M.; Duda J. L., \& Ntoumanis, N. (2004). Dimensions of Coaching Behavior, Need Satisfaction, and the Psychological and Physical Welfare of Young Athletes. Motivation and Emotion, 28(3), 297-313.

Ryan, R. M., \& Deci, E. L. (2000). Self-determination theory and the facilitation of intrinsic motivation, social development and well-being. American Psychologist, $55(1), 68-78$.

Sarrazin, P. (2001). Approches socio-cognitives de la motivation appliqués au champ du sport et de l'Éducation Physique et Sportive. Diploma de Estudios Avanzados, Universidad Joseph Fourier, Grenoble, France. 
Sánchez-Oliva, D.; Leo, F. M.; Sánchez-Miguel, P. A.; Amado, D.; García-Calvo, T. (2010). Relación del clima motivacional creado por el entrenador con la motivación autodeterminada y la implicación hacia la práctica deportiva. Revista Internacional de Ciencias del Deporte, 20(6), 177-195.

http://www.cafyd.com/REVISTA/02001.pdf

Sarrazin, P.; Vallerand, R.; Guillet, E.; Pelletier, L., \& Cury, F. (2002). Motivation and dropout in female handballers: A 21-month prospective study. European Journal of Social Psychology, 32, 395-418.

Sousa, C.; Arevalo, A.; Ramis, S.; Torregrosa, M.; Viches, D., \& Cruz, J. (2006). Design and implementation of coach training program in youth soccer. En Resúmenes del $26^{\circ}$ Congreso Internacional de Psicología Aplicada (p. 984). Grecia: Asociación Internacional de Psicología Aplicada.

Standage, M.; Duda, J. L., \& Ntoumanis, N. (2003). Predicting motivational regulations in physical education: The interplay between dispositional goal orientations, motivational climate and perceived competence. Journal of Sports Sciences, 21, 631-647.

Standage, M.; Duda, J. L., \& Pensgaard, A. M. (2005). The Effect of Competitive Outcome and Task-Involving, Ego-Involving, and Cooperative Structures on the Psychological Well-Being of Individuals Engaged in a Co-Ordination Task: A SelfDetermination Approach. Motivation and Emotion, 29(1), 41-68.

Theodosiou, A.; Mantis, K., \& Papaioannou, A. (2008). Student self-reports of metacognitive activity in physical education classes. Age-group differences and the effect of goal orientations and perceived motivational climate. Educational Research and Review, 3(12), 353-364.

Torregrosa, M.; Cruz, J.; Sousa, C.; Vildarich, C.; Villamarín, F.; Garcia-Mas, A., y Palou, P. (2007). La influencia de padres y madres en el compromiso deportivo de futbolistas jóvenes. Revista Latinoamericana de Psicología, 39, 227-237.

Vallerand, R. J. (2001). A hierarchical model of intrinsic and motivation in sport and exercise. En G.C. Roberts (Ed.), Advances in motivation in sport and exercise (pp. 263-320). Champaign, IL: Human Kinetics.

Vallerand, R. J. (2007). A hierarchical model of intrinsic and extrinsic motivation for sport and physical activity. En M. S. Hagger y N. L. D. Chatzisarantis (Eds.), Intrinsic motivation and self-determination in exercise and sport (pp. 255-279). Champaign: Human Kinetics.

Vallerand, R. J.; Brière, N. M.; Blanchard, C., \& Provencher, P. (1997). Development and validation of the multidimensional sportspersonship orientations scale. Journal of Sport and Exercise Psychology, 16, 126-140. 